Communications in Physics, Vol. 25, No. 3 (2015), pp. 211-217

DOI:10.15625/0868-3166/25/3/6523

\title{
SUPERHEAVY DARK MATTER IN THE SUPERSYMMETRIC ECONOMICAL 3-3-1 MODEL
}

\author{
DO THI HUONG \\ Institute of Physics, Vietnam Academy of Science and Technology, \\ 10 Dao Tan, Ba Dinh, Hanoi, Vietnam \\ E-mail: dthuong@iop.vast.ac.vn \\ Received 17 July 2015 \\ Accepted for publication 12 September 2015
}

\begin{abstract}
We suggest the supersymmetric particles contained in the supersymmetric economical 3-3-1 model were produced during the heating time. They have not been thermodynamic equilibrium when it froze out. It leads to the lightest supersymmetric particle can be a good candidate for superheavy dark matter. With assumption the lightest supersymmetric particle is Bino, we show that the correct contribution of the superheavy dark matter (SHDM) species to the present critical density requires the Bino mass is order $10^{12} \mathrm{GeV}$ and there is not exists a large mass hierarchy of the superpartner in the considered model.
\end{abstract}

Keywords: supersymmetric, superheavy dark matter.

There is decided evidence that the dominant component of the matter density in the universe is dark. The most interesting evidence of the existence of dark matter (DM) is the observation of flat rotation for spiral galaxies [2]. Recent experiments (WMAP and PLANCK) have shown [1] a large portion of the total mass-energy of the universe such as dark matter is about 25 percents and 70 percents of dark energy. However, the standard model (SM) fails to prove the origin of DM. So we have to go beyond the SM.

In the standard dark matter scenarios, the weak interaction massive particles (WIMPs) are usually considered to have once been in local thermodynamic equilibrium (LTE) and their present abundance depends on their self-annihilation cross section. The largest possible cross section is roughly $M_{D M}^{-2}$. This implies that very massive WIMPs would have such a small annihilation cross section that their present abundance would be too large. Hence, the maximum mass for thermal WIMPs is expected to be a few hundred TeV [3].

While a thermal origin for WIMPs is the most common assumption, it is not the simplest possibility. Recently, it has been pointed out that DM particles never attain LTE in the past and their mass may be in range $10^{12}$ to $10^{19} \mathrm{GeV}[4,5,23]$. There are a number cosmological production mechanisms for nonthermal SHDM. This was discussed more detail in [5]. It is important 
to realized that the energy density of non relativistic particles decreases slower than one of radiation. Their relic abundance increases by factor $\frac{a\left(t_{o}\right)}{a\left(t_{*}\right)}$ with respect to radiation, where $a\left(t_{o}\right), a\left(t_{*}\right)$ are respectively the scale factors of the universe today and at the epoch of particle produced. If massive particles are produced at the early time, i.e. during inflation or reheating time, the factor $\frac{a\left(t_{o}\right)}{a\left(t_{*}\right)} \simeq 10^{22}$. Hence, it is not surprising if a small energy fraction of inflaton can be conversed to the SHDM by several mechanisms, such as thermal production at reheating [4,23], the nonperturbative regime of a broad parametric resonance at the preheating [7], production by topological defects [8].

At present, the most interesting manifestation of SHDM may be the observed ultra-high energy cosmic rays (UHECR) [14]. There are three basic signatures of UHECRs from SHDM those are listed in [13], namely: The UHECR flux from SHDM has no GZK cutoff in the energy spectrum. The SHDMs decay or annihilate dominantly to the pion then UHE neutrinos and photons are dominant component of the primary flux. An anisotropic UHECR flux from SHDM that explains why the Sun is not central position in the galactic halo. For more detail of the status of SHDM, the interested reader can see in [13].

There are two necessary conditions for SHDM candidates. First, the SHDM have to be stable. This may result from the supersymmetric theory [9], the theories with discrete gauge symmetries [10] and the string theory and M-theory [11]. In particularly if R parity is conserved, the lightest supersymmetric particle (LSP) is a promising DM candidate. The second condition for SHDM is that the particle must not have been in equilibrium when it froze out.

In fact there are many attractive properties of low scale SUSY (1 TeV) such as three couplings meet in the minimal supersymmetric standard model (MSSM); the fine-turning problem of the SM Higgs is naturally remedied in MSSM; the thermal relic dark matter requires the SUSY breaking scale is below to the $\mathrm{TeV}$ scale. However, these attractive properties of the low scale SUSY are overshadowed by several less appealing future [12]. Therefore, split SUSY have been considered to avoid the problem of low scale SUSY [24]. In this theory the mass spectrum of SUSY particle is separated in two parts: One is kept at TeV and other one is closed at the GUT scale.

In this work, we go one step further by assuming that SUSY is broken at the large scale. The LSP can be produced nonthermally at the end of inflation. Hence the the LSP is stale and very massive. If LHC can not detect the supersymmetric particles at low energy scale, our assumption may be very interested to connect SUSY to physical world. Especially we concentrate on the supersymmetric economical 3-3-1 model. The 3-3-1 models are the extended SM model based the extension of the gauge group. This class of model can explain the number of fermion families [15], the hierarchical heaviness of top quark [16], the strong CP conservation [17], and the electric charges quantization [18]. One of the weaknesses of the mentioned 3-3-1 models that reduces scalar sectors. The attempt on this direction to realize simpler scalar sectors is the recently constructed 3-3-1 model with minimal Higgs sector called the economical 3-3-1 model $[19,20]$.The supersymmetric version of the economical model was constructed in [21]. The thermal DM have been studied in [22]. We assume that the lightest neutralino $\chi \equiv \chi_{1}$ is the lightest of the supersymmetric particles. The neutralino mass matrix $M_{\chi}$ in the 
basis $\widetilde{\psi}^{o}=\left(\widetilde{\chi_{1}^{o}}, \widetilde{\chi_{1}^{o \prime}}, \widetilde{\chi_{2}^{o}}, \widetilde{\chi_{2}^{o \prime}}, \widetilde{\rho_{1}^{o}}, \widetilde{\rho_{1}^{o \prime}}, \widetilde{\mathscr{B}}, \widetilde{\mathscr{W}_{3}}, \widetilde{\mathscr{W}} 8, \widetilde{\mathscr{X}}=\frac{\widetilde{\mathscr{W}}_{4}+i \widetilde{\mathscr{W}}_{5}}{2}, \widetilde{\mathscr{X}^{*}}=\frac{\widetilde{\mathscr{W}}_{4}-i \widetilde{\mathscr{W}_{5}}}{2}\right)$ is given in [22]

$$
M_{\widetilde{N}}=\left(\begin{array}{ccccccccccc}
0 & -\mu_{\chi} & 0 & 0 & 0 & 0 & -\frac{g^{\prime} u}{3 \sqrt{6}} & \frac{g u}{2} & \frac{g u}{2 \sqrt{3}} & \frac{g w}{\sqrt{2}} & 0 \\
-\mu_{\chi} & 0 & 0 & 0 & 0 & 0 & \frac{g^{\prime} u^{\prime}}{3 \sqrt{6}} & \frac{g u^{\prime}}{2} & \frac{g u^{\prime}}{2 \sqrt{3}} & \frac{g w^{\prime}}{\sqrt{2}} & 0 \\
0 & 0 & 0 & & -\mu_{\chi} & 0 & -\frac{g^{\prime} w}{3 \sqrt{6}} & 0 & -\frac{g w}{\sqrt{3}} & 0 & \frac{g u}{\sqrt{2}} \\
0 & 0 & -\mu_{\chi} & 0 & 0 & 0 & \frac{g^{\prime} w^{\prime}}{3 \sqrt{6}} & 0 & -\frac{g w^{\prime}}{\sqrt{3}} & 0 & \frac{g u^{\prime}}{\sqrt{2}} \\
0 & 0 & 0 & 0 & 0 & -\mu_{\rho} & \frac{2 g^{\prime} v}{3 \sqrt{6}} & -\frac{g v}{2} & \frac{g v}{2 \sqrt{3}} & 0 & 0 \\
0 & 0 & 0 & 0 & -\mu_{\rho} & 0 & -\frac{2 g^{\prime} v^{\prime}}{3 \sqrt{6}} & -\frac{g v^{\prime}}{2} & \frac{g v^{\prime}}{2 \sqrt{3}} & 0 & 0 \\
-\frac{g^{\prime} u}{3 \sqrt{6}} & \frac{g^{\prime} u^{\prime}}{3 \sqrt{6}} & -\frac{g^{\prime} w}{3 \sqrt{6}} & \frac{g^{\prime} w^{\prime}}{3 \sqrt{6}} & \frac{2 g^{\prime} v}{3 \sqrt{6}} & -\frac{2 g^{\prime} v^{\prime}}{3 \sqrt{6}} & \mathscr{M}_{\mathscr{B}} & 0 & 0 & 0 & 0 \\
\frac{g u}{2} & \frac{g u^{\prime}}{2} & 0 & 0 & -\frac{g v}{2} & -\frac{g v^{\prime}}{2} & 0 & \mathscr{M}_{3} & 0 & 0 & 0 \\
\frac{g u}{2 \sqrt{3}} & \frac{g u^{\prime}}{2 \sqrt{3}} & -\frac{g w}{\sqrt{3}} & -\frac{g w^{\prime}}{\sqrt{3}} & \frac{g v}{2 \sqrt{3}} & \frac{g v^{\prime}}{2 \sqrt{3}} & 0 & 0 & \mathscr{M}_{8} & 0 & 0 \\
\frac{g w}{2} & \frac{g w^{\prime}}{2} & 0 & 0 & 0 & 0 & 0 & 0 & 0 & \mathscr{M}_{45} & 0 \\
0 & 0 & \frac{g u}{2} & \frac{g u^{\prime}}{2} & 0 & 0 & 0 & 0 & 0 & 0 & \mathscr{M}_{45}
\end{array}\right)
$$

where $\mathscr{M}_{4}=\mathscr{M}_{5} \equiv \mathscr{M}_{45}$. In the limit

$$
\begin{aligned}
& v, v^{\prime}, u, u^{\prime}, w, w^{\prime} \ll\left|\mu_{\rho}-\mathscr{M}_{\mathscr{B}}\right|,\left|\mu_{\rho}-\mathscr{M}_{3}\right|,\left|\mu_{\rho}-\mathscr{M}_{8}\right|,\left|\mu_{\rho}-\mathscr{M}_{45}\right| \\
& v, v^{\prime}, u, u^{\prime}, w, w^{\prime} \ll\left|\mu_{\chi}-\mathscr{M}_{\mathscr{B}}\right|,\left|\mu_{\chi}-\mathscr{M}_{3}\right|,\left|\mu_{\chi}-\mathscr{M}_{8}\right|,\left|\mu_{\chi}-\mathscr{M}_{45}\right|,
\end{aligned}
$$

we can use a perturbation theory and at the leading order the mass eigenstates are give in [22] such as

$$
\begin{aligned}
& \widetilde{\chi}_{1}=\widetilde{\mathscr{B}}, \widetilde{\chi}_{2}=\widetilde{\mathscr{W}_{3}}, \widetilde{\chi}_{3}=\widetilde{\mathscr{W}}, \widetilde{\chi}_{4}=\widetilde{\mathscr{X}^{*}}, \widetilde{\chi}_{5}=\widetilde{\mathscr{X}} \\
& \widetilde{\chi}_{6}, \widetilde{\chi}_{7}=\frac{\widetilde{\rho}^{o} \pm \widetilde{\rho_{1}^{\prime o}}}{\sqrt{2}}, \widetilde{\chi}_{8}, \widetilde{\chi}_{9}=\frac{\widetilde{\chi^{o}}{ }_{1} \pm \widetilde{\chi_{1}^{o l}}}{\sqrt{2}}, \widetilde{\chi}_{10}, \widetilde{\chi}_{11}=\frac{\widetilde{\chi^{o}}{ }_{2} \pm \widetilde{\chi_{2}^{\prime o}}}{\sqrt{2}}
\end{aligned}
$$

The mass values depend on the parameters space. If $\mathscr{M}_{\mathscr{B}}<\mathscr{M}_{3}<\mathscr{M}_{8}<\mathscr{M}_{45} \ll \mu_{\chi}, \mu_{\rho}$, the LSP is a Bino-like $\widetilde{\chi}_{1}$. Let us assume that the Bino have not been in equilibrium when it froze out. There are mechanisms that can produce massive particles. In this work, we assume that the Bino was produced during reheating. As Ref. [23] at the end of inflation, the inflaton energy density is converted to radiation known as reheating. However, a small fraction of the inflaton energy can be converted to produce massive particle. The particles with mass greater than reheating temperature can be created during the reheating time. The present contribution of the SHDM species to the critical density is given in [23] as follows

$$
\Omega_{\widetilde{\chi}_{1}} h^{2}=M_{\widetilde{\chi}_{1}}^{2}<\sigma|v|>\left(\frac{g_{*}}{200}\right)^{\frac{-3}{2}}\left(\frac{2000 T_{R H}}{M_{\widetilde{\chi}_{1}}}\right)^{7},
$$

where $g_{*}$ is the number of effective degrees of the radiation energy density, $<\sigma|v|>$ is the thermal average of the $\widetilde{\chi}_{1}$ annihilation cross section and $T_{R H}$ is the reheat temperature. According to [24], the thermal cross section have been written as

$$
<\sigma v_{r e l}>=\widetilde{a}+\widetilde{b} x
$$


where

$$
\begin{aligned}
& \widetilde{a}=\sum_{f} \theta\left(m_{\chi_{1}}-m_{f}\right) \frac{p}{m_{\chi_{1}}} m_{f}^{2}\left(A_{f}-B_{f}\right)^{2}, \\
& \widetilde{b}=\sum_{f} \theta\left(m_{\chi_{1}}-m_{f}\right) \frac{1}{2 \pi} \frac{p}{m_{\chi_{1}}}\left(\left(A_{f}^{2}+B_{f}^{2}\right)\left(4 m_{\chi_{1}}^{2}-m_{f}^{2}\right)+6 A_{f} B_{f} m_{f}^{2}\right),
\end{aligned}
$$

with $p=\sqrt{m_{\chi_{1}}^{2}-m_{f}^{2}}$.

Let us consider a thermal average cross section of the $\mathrm{X}$ annihilation. We assume that the most dominated annihilation channels are $\tilde{\chi}_{1} \widetilde{\chi}_{1} \rightarrow \widetilde{f} f,(f=q, l, v)$. The Feynman diagrams for Bino annihilation processes are depicted in the Fig. 1. With this assumption, the coefficients $A_{f}, B_{f}$ have a form as follows

$$
\begin{gathered}
A_{f}=\frac{Y_{f_{L}}^{2} g^{\prime 2}}{12 m_{\tilde{f}_{L}}^{2}}-\frac{Y_{f_{R}}^{2} g^{\prime 2}}{12 m_{\tilde{f}_{R}}^{2}}, \\
B_{f}=-\frac{Y_{f_{L}}^{2} g^{\prime 2}}{12 m_{\tilde{f}_{L}}^{2}}-\frac{Y_{f_{R}}^{2} g^{\prime 2}}{12 m_{\tilde{f}_{R}}^{2}},
\end{gathered}
$$

where $Y_{L}, Y_{R}$ are hypercharge of left- and right-handed ordinary quark and lepton.

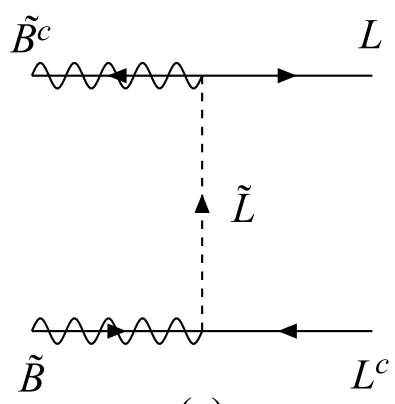

(a)

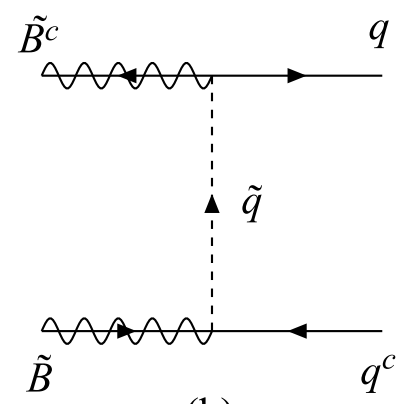

(b)

Fig. 1. Feynman diagrams contributing to annihilation of Bino dark matter

Let us now turn to the numerical analysis of the contribution of the SHDM to the present DM density. For this purpose, we assume that $g^{\prime}=0.6, g_{\star}=150, x_{f} \simeq \frac{1}{20}$. The gravitino production requires that the reheating temperature $T_{R} \in\left[10^{9}, 10^{10}\right] \mathrm{GeV}$. Hence, we fix $T_{R}=10^{9} \mathrm{GeV}$.

Fig. 2 illustrates the present contribution of the SHDM species to the critical density by taking $m_{\widetilde{f}_{L}}=m_{\widetilde{f}_{R}}=m_{\chi_{1}}$. The result shows that the present SHDM density decreases rapidly if the SHDM mass increase, namely: If we take the limit on the mass of $\widetilde{\chi}_{1}: m_{\tilde{\chi}_{1}}<8.5 \times 10^{11} \mathrm{GeV}$, we obtain the SHDM relic density is too large to compare to the WMAP data [1] $\Omega_{D M} h^{2} \simeq 0.11$ and if the SHDM mass: $m_{\chi_{1}}>10^{12} \mathrm{GeV}$, their relic density is too small to compare with the WMAP data. It means that to match the WMAP data, the limit on the SHDM mass is: $\left[8.5 \times 10^{11}, 10^{12}\right]$ 
$\mathrm{GeV}$. These values are as large as $10^{3}$ times the reheating temperature. Our results are entirely consistent with the predicted results given in [23].

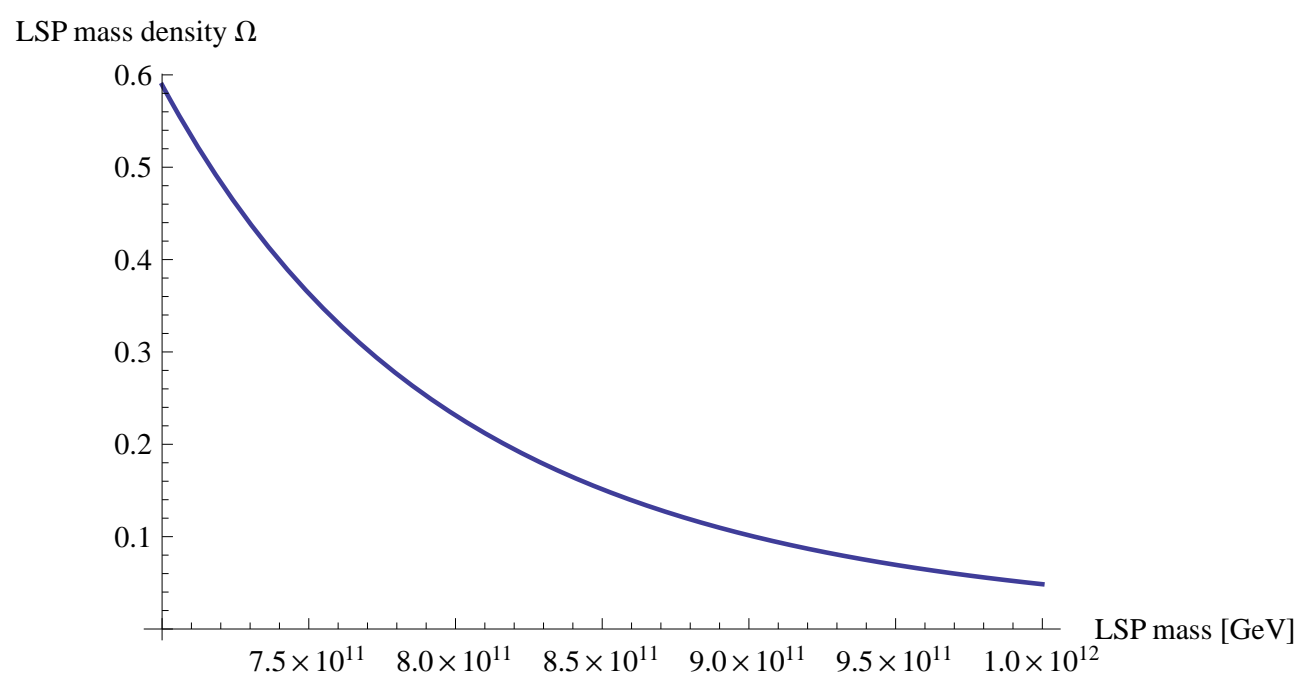

Fig. 2. The present contribution of the SHDM species to the critical density as a function of their mass with the condition: $m_{\widetilde{f}_{L}}=m_{\widetilde{f_{R}}}=m_{\chi_{1}}$

The results given in Fig. 3 are plotted for $m_{\widetilde{l}_{L}}=m_{\widetilde{l}_{R}}=10^{2} \times m_{\chi_{1}}$ while the results given in Fig.4 are plotted for $m_{\tilde{l}_{L}}=m_{\tilde{l}_{R}}=10^{4} \times m_{\chi_{1}}$. If the slepton mass is more heavier than that of the SHDM, the SHDM will become the less heavier. In particularly, if the values of slepton mass are $10^{4}$ times as heavy as those of the SHDM, in order to address to the WMAP data, we obtain the limit on the values of the SHDM mass is the same order as the reheating temperature. This is contrary to results indicated in [23].

LSP mass density $\Omega \mathrm{h}^{\wedge} 2$

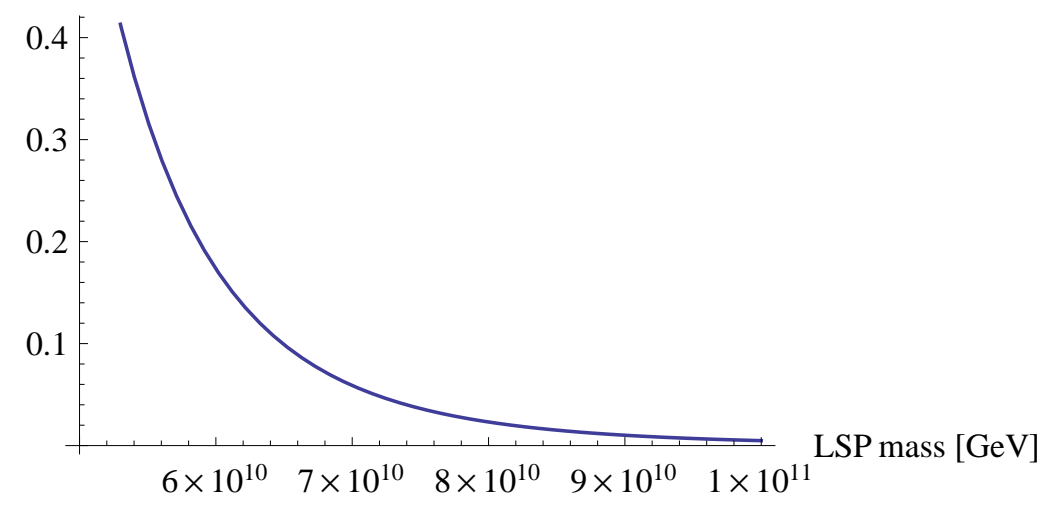

Fig. 3. The present contribution of the SHDM species to the critical density as a function of their mass with the condition: $m_{\widetilde{f}_{L}}=m_{\widetilde{f_{R}}}=10^{2} m_{\chi_{1}}$ 
LSP mass density $\Omega \mathrm{h}^{\wedge} 2$

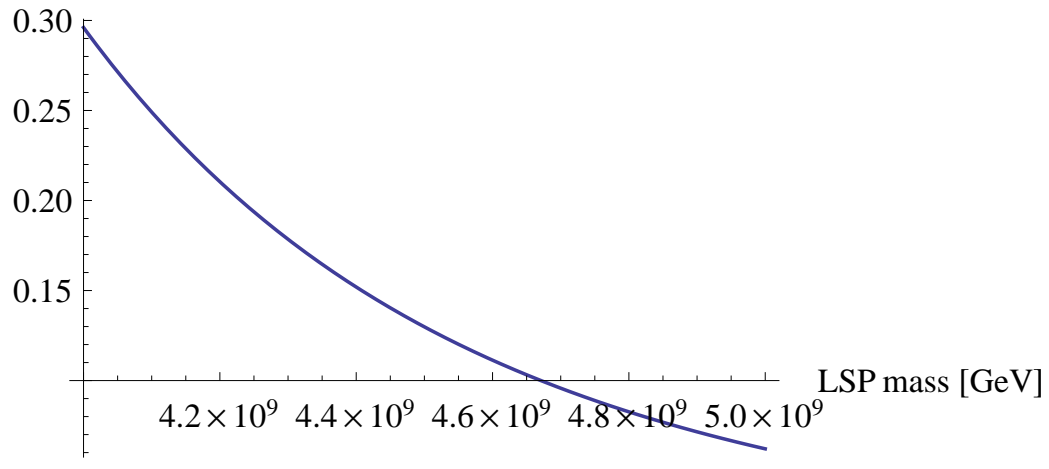

Fig. 4. The present contribution of the SHDM species to the critical density as a function of their mass with the condition $m_{\tilde{f}_{L}}=m_{\widetilde{f_{R}}}=10^{4} m_{\chi_{1}}$

We conclude this paper with following remarks: We have assumed that the SUSY is broken at the very high scale. Hence, the LSP is suggested as a suitable candidate for the SHDM. The requirement that the LSP is Bino and produced during reheating time with correct abundance. If the SHDM gives the main contribution to the DM abundance, the SHDM mass is not larger than $10^{12} \mathrm{GeV}$ with the reheating temperature fixed at $10^{9} \mathrm{GeV}$. From the numerical results, we show that there is not exists a large mass hierarchy of the superpartner in the considered theory.

\section{ACKNOWLEDGMENT}

This research is funded by Vietnam National Foundation for Science and Technology Development (NAFOSTED) under grant number 103.01-2014.69.

\section{REFERENCES}

[1] K. A. Olive et al. (Particle Data Group), Chin. Phys. C 38 (2014) 090001.

[2] G. Jungman, M. Kamionkowski, and K. Greist, Phys. Rep. 267 (1995) 196.

[3] K. Greist and M. Kamionkowski, Phys. Rev. Lett. 64 (1990) 615.

[4] V. Berezinsky, M. Kachelrieß, and A. Vilenkin, Phys. Rev. Lett. 79 (1997) 4302.

[5] Daniel J. H. Chung, Edward W. Kolb, Antonio Riotto, Phys. Rev. Lett. 81 (1998) 4048-4051.

[6] Daniel J. H. Chung, Edward W. Kolb, Antonio, Phys. Rev. D 60 (1999) 063504.

[7] E. W. Kolb, A. Notari, and A. Riotto, Phys. Rev. D 68 (2003) 123505; L . Kofman, A. D. Linde and Starobinsky, Phys. Rev. Lett. 73 (1994) 3195; G. N. Felder, L. Kofman, and A. D. Linde, Phys. Rev. D 59 (1999) 123523.

[8] E. W. Kolb, D. J. H. Chung, and A. Riotto, arXiv: hep-ph/9810361; D. J. H. Chung, E. W. Kolb, and A. Riotto, Phys. Rev. D 59 (1998) 023501;

[9] S. Raby, Phys. Rev. D 56 (1997) 2852.

[10] K. Hamaguchi, Y. Nomura, and T. Yanagida, hep-ph/9805346.

[11] K. Benakli, J. Ellis, and D. V. Nanopoulos, hep-ph/9803333.

[12] J. R. Ellis, K. Enqvist, D. V. Nanopoulos, and F. Zwirner, Nucl. Phys. B 276 (1986) 14; R. Barbieri and G. F. Giudice, Nucl. Phys. B 306 (1988) 63; V. Berezinsky et al., Astropart. Phys. 5 (1996) 1; J. R. Ellis, K. A. Olive, Y. Santoso, and V. C. Spanos, Phys. Lett. B 565 (2003) 176.

[13] R. Aloisio, V. Berezinsky, M. Kachelriess, Phys. Rev. D 74 (2006) 023516.

[14] V. Berezinsky, M. Kachelrie and A. Vilenkin, Phys. Rev. Lett. 79 (1997) 4302 [astro-ph/9708217]; S. L. Dubovsky and P. G. Tinyakov, JETP Lett. 68 (1998) 107 [hep-ph/9802382]; 
[15] P. H. Frampton, Phys. Rev. Lett. 69 (1992) 2889;

[16] D. G. Dumm, F. Pisano, and V. Pleitez, Mod. Phys. Lett. A 9 (1994) 1609; H. N. Long and V. T. Van, J. Phys. G 25 (1999) 2319;

[17] P. B. Pal, Phys. Rev. D 52 (1995) 1659;

[18] F. Pisano, Mod. Phys. Lett A 11, 2639 (1996); A. Doff and F. Pisano, Mod. Phys. Lett. A 14 (1999) 1133; C. A. de S. Pires and O. P. Ravinez, Phys. Rev. D 58 (1998) 035008.

[19] W. A. Ponce, Y. Giraldo and L. A. Sanchez, Phys. Rev. D 67 (2003) 075001

[20] P. V. Dong, H. N. Long, D. T. Nhung and D. V. Soa, Phys. Rev. D 73 (2006) 035004; P. V. Dong, D. T. Huong, Tr. T. Huong and H. N. Long, Phys. Rev. D 74 (2006) 053003; P. V. Dong, H. T. Hung and H. N. Long, Phys. Rev. D 86 (2012) 033002, for a review, see: P. V. Dong and H. N. Long, Adv. High Energy Phys. 2008 (2008) 739492, [arXiv:0804. 3239(hep-ph)].

[21] P. V. Dong, D. T. Huong, M. C. Rodriguez and H. N. Long, Nucl. Phys. B 772 (2007) 150 .

[22] D. T. Huong, H. N. Long, JHEP 0807 (2008) 049.

[23] Daniel J. H. Chung, Edward W. Kolb, Antonio, Phys. Rev. D 60 (1999) 063504.

[24] J. Ellis et al., Nucl. Phys. B 238 (1984) 453. 